\title{
La teoría de la definición en Leibniz, Spinoza y Tschirnhaus
}

\author{
MARIO A. NARVÁEZ \\ Facultad de Ciencias Humanas-UNLPam
}

DOI: $10.36446 /$ rlf2020202

\begin{abstract}
Resumen: Leibniz, Spinoza yTschirnhaus intercambiaron opiniones sobre diversas cuestiones filosóficas, entre ellas la problemática en torno a la naturaleza del método y de la definición perfecta. Sin embargo, los estudios sobre la relación entre estos filósofos generalmente desatendieron la cuestión metodológica. En el presente trabajo nos ocuparemos de hacer un aporte tendiente a llenar ese vacío, examinando los resultados del intercambio mencionado en los proyectos metodológicos de los tres filósofos. En particular enfocamos el análisis sobre la teoría de la definición, puesto que es el punto clave de las concepciones metodológicas de los tres filósofos. Intentaremos mostrar que Spinoza, al combinar elementos metodológicos de Hobbes con elementos epistemológicos cartesianos, ejerció una importante influencia sobre Leibniz y Tschirnhaus acerca de cómo debería ser la definición perfecta y más adecuada para el método cuyo objetivo es alcanzar la verdad. En vistas de este objetivo, realizaremos un examen comparativo centrado en la función que le
\end{abstract}


asignan a la definición en la estructura metodológica, así como también en el modo en que cada uno concibe la naturaleza de la misma.

Palabras claves: definición genética, método, conocimiento, verdad.

\title{
The Theory of Definition in Spinoza, Leibniz and Tschirnhaus
}

\begin{abstract}
Leibniz, Spinoza and Tschirnhaus exchanged opinions on various philosophical questions, among them the problematic about the nature of the method and the perfect definition. However, studies on the relationship between these philosophers generally ignored the methodological question. In the present work we will make a contribution to fill that gap, examining the results of the exchange mentioned in the methodological projects of the three philosophers. In particular, we focus on the analysis of the theory of definition, since it is the key point of the methodological conceptions of the three philosophers. We will try to show that Spinoza, by combining Hobbes' methodological elements with Cartesian epistemological elements, exerted an important influence on Leibniz and Tschirnhaus about what the perfect and most suitable definition should be for the method whose objective is to reach the truth. In view of this objective, we will carry out a comparative examination focused on the function assigned to the definition in the methodological structure, as well as in the way in which each one conceives its nature.
\end{abstract}

Key-words: genetic definition, method, knowledge, truth.

\section{Introducción}

T a existencia de un intercambio intelectual entre Leibniz, TschirLnhaus y Spinoza es bien conocida. Es sabido que Leibniz se interesó por la filosofia de Spinoza, que se entrevistó con él y que discutió personalmente muchas de las teorías de la Ética con el amigo en común Tschirnhaus. Este, por su parte, mantuvo un intercambio más fluido con Spinoza, tanto personal como epistolar, y la influencia puede notarse en varias de sus ideas publicadas en la Medicina Mentis. Sin embargo, los análisis de este intercambio comúnmente pasan por alto el tema del método y en particular de la definición. Esto último constituye una carencia importante, pues el interés de Leibniz por Spinoza también estuvo dirigido hacia esos 
temas y quizá haya reflexionado sobre estas cuestiones personalmente con Tschirnhaus. De hecho en la famosa carta 82 de Tschirnhaus, fechada en Paris el 23 de junio de 1676 (Spinoza, Ep. 82, G IV 333-334) ${ }^{1}$, en la cual varios historiadores han visto la presencia de Leibniz detrás del interés en el tema de la extensión ${ }^{2}$, Tschirnhaus interroga a Spinoza por el modo en que pueden deducirse las propiedades de una cosa a través de su definición. En respuesta a esa carta, Spinoza recuerda algo similar a lo que ya le había mencionado en una carta anterior, esto es, que de la definición de Dios (definición causal) pueden deducirse todas las propiedades de lo definido. Es dificil pensar que, en el marco de estas reflexiones, Tschirnhaus no haya discutido con Leibniz el modo en que Spinoza entiende la definición causal (Laerke 2008: 365 y ss.). Con más razón teniendo en cuenta que para los tres filósofos la cuestión metodológica era un asunto de suma importancia. Además, es posible también que Leibniz haya tratado el tema en la entrevista personal que mantuvo con Spinoza en Holanda, sobre la que poco se sabe con certeza. Como quiera que sea, no hay dudas de que tanto Tschirnhaus como Leibniz conocían las ideas de Spinoza y su puesta en práctica en el método de la filosofia.

Así pues, dado que, como veremos luego, ambos filósofos alemanes defendieron concepciones metodológicas en las que la definición causal ocupa un lugar preponderante, cabe preguntarse hasta qué punto dichas concepciones de la definición no son deudoras de la concepción spinoziana. En este sentido, lo que nos proponemos en el siguiente trabajo es establecer qué quedó de ese intercambio con Spinoza. Si bien nos centraremos en la teoría de la definición, ya que para los tres es el componente fundamental del método, para desarrollar esa temática es preciso tener en cuenta algunas ideas metodológicas generales.

Intentaremos mostrar que aunque no se pueda hablar de una influencia completa de Spinoza, pues, por un lado, no adoptan su posición en un todo, y por otro, la concepción de Spinoza proviene en cierto modo de Hobbes, sí se podría decir que Spinoza permite a Tschirnhaus y a Leibniz conectar la teoría de la definición hobbesiana con los aspectos fundamentales de la epistemología cartesiana, esto es, con la teoría de las ideas tal como

\footnotetext{
${ }^{1}$ Para las citas de Spinoza utilizaremos las siguientes abreviaturas: Ep. (Épistola), TIE (Tratado de la Reforma del Entendimiento), E (Ética), seguido del libro en números romanos, p (proposición), def. (definición), esc. (escolio), dem. (demostración), según corresponda. Las citas remiten a la edición de Gebhardt (G, seguido de volumen y número de página) y para todas las citas textuales, las traducciones al español de Atilano Domínguez.

${ }^{2}$ Véanse Goldenbaum 2014: 35 y Kulstad 2014: 51 y ss.;
} 
cada uno de ellos la reelabora. En este sentido se puede decir que tanto uno como otro siguen a Spinoza. Por otra parte, podremos ver que la reelaboración de la teoría de las ideas que cada uno llevó a cabo, determina también las diferencias en la concepción de la definición.

\section{La definición y el método}

Comenzaremos examinado cuál es la función que los tres filósofos le atribuyen a la definición y qué lugar ocupa en la concepción metodológica que cada uno sostiene. Pues bien, en el ambiente intelectual de la modernidad, tal como lo refleja la famosa Lógica de Port Royal, parece haber dos maneras básicas de entender la definición. O bien, como un punto de partida, esto es, como medio de evitar la confusión que produce el lenguaje, fijando de antemano el uso de los términos; o bien, como un punto de llegada, esto es, como una proposición que se obtiene a partir de un proceso demostrativo. El primer tipo de definición es llamada "definición nominal", el segundo "definición de cosa". Las primeras no pueden ser impugnadas dado que son meras estipulaciones acerca del uso de los nombres; por ello, pueden ser tomadas como principios de la ciencia. Las segundas, en cambio, se refieren a la esencia de una cosa, de modo que deben ser probadas (Arnauld y Nicole Logique, 81-82).

A continuación veremos que Spinoza, Leibniz y Tschirnhaus desafian en cierto modo esta clasificación. Si bien mantienen algunos aspectos de la misma, otros son arrojados por la borda. Ciertamente, están de acuerdo en que las definiciones deben ser el punto de partida, el punto de apoyo sobre el cual se eleva el edificio del conocimiento. Por ello, como veremos, sus metodologías giran en torno a la definición. No obstante, en la manera de explicar la naturaleza de la definición perfecta se van a apartar de Port Royal. Para comenzar, consideraremos dos breves pasajes del Tratado de la Reforma del Entendimiento, en los que Spinoza aclara esquemáticamente cuál es la función de la definición y qué lugar ocupa en la estructura metodológica. Según el primero de ellos,

La vía correcta de la investigación consiste [...] en formar los pensamientos a partir de una definición dada (recta inveniendi via est ex data aliqua definitione cogitationes formare); y resultará tanto más fácil y eficaz, cuanto mejor hayamos definido la cosa (Spinoza, TIE \ 94, G II 34).

Y un poco más adelante agrega: 
El concepto o definición de la cosa debe ser tal que, considerada en sí sola y no unida a otras, se puedan concluir de ella todas sus propiedades... (Spinoza TIE $₫ 96$ G II 35 y $₫ 97$, G II 35-36)

Como puede verse aquí, la definición se ubica como el punto de partida de un procedimiento orientado a descubrir nuevas verdades. Pero Spinoza no concibe un paso ulterior de fundamentación, con lo cual es de suponer que el descubrimiento y la fundamentación son, por así decir, dos aspectos de un mismo proceso. En efecto, no hay indicios en el TIE de la conocida escisión entre el ars inveniendi y el ars judicandi, que aparece, como señala Arndt, en otros proyectos metodológicos del siglo XVII (Arndt 1971: 4-7). Es cierto que Spinoza dedica unos cuantos parágrafos de su tratado a distinguir la idea verdadera de la falsa, procedimiento indispensable para poder definir correctamente. Dicho procedimiento podría remitir al método del análisis ${ }^{3}$ y podríamos pensar que estamos en presencia del ars inveniendi. Sin embargo, esta lectura contradiría el pasaje citado, en el cual Spinoza afirma claramente que la vía de descubrimiento -o, literalmente, de la invención ${ }^{4}$ - comienza con las definiciones, y con la deducción de las propiedades a partir de dichas definiciones. Por lo tanto, dicha vía es claramente sintética.

Tschirnhaus, por su parte, parece mantener una visión muy similar respecto de la función asignada a la definición. En un pasaje que podemos considerar como una brevísima síntesis de su método para encontrar la verdad afirma lo siguiente:

[...] yo ordenaría de entrada todos los conceptos primeros posibles de los cuales los otros están formados y los llamaré a continuación "definiciones"; en segundo lugar, consideraré separadamente esas mismas definiciones y llamaré "axiomas" a las propiedades que surgen de allí; en tercer lugar, pondré en relación unas con otras a las definiciones de todas las maneras posibles y designaré con el nombre de "teoremas" a las verdades así obtenidas. (Tschirnhaus $M M, 92)^{5}$

\footnotetext{
${ }^{3}$ Veánse Joachim 1901: 12 y De Angelis 1968: 43-47

${ }^{4}$ Mantendremos aquí la traducción más común de inventio por "descubrimiento" ya que consideramos que el término "invención” está más asociado en castellano a la creación de dispositivos tecnológicos que al hallazgo de proposiciones verdaderas (empíricas o de otro tipo). ${ }^{5}$ Las citas de la Medicina Mentis reproducen nuestra traducción de la traducción francesa de J.-P. Wurtz, por ello indicamos las páginas de su edición y precedida de la abreviatura $M M$.
} 
La definición, como puede verse, es el punto de partida. De ella se obtienen las propiedades de los objetos definidos, es decir, lo que nuestro autor denomina "axiomas". Luego, de la vinculación entre las definiciones entre sí surgen los llamados "teoremas". No es necesario entrar aquí en la cuestión de cuál es la naturaleza de los axiomas y los teoremas. Salta a la vista que se trata de un procedimiento sintético en el cual se unen estrechamente el descubrimiento de nuevas verdades y la fundamentación de las mismas. Es importante destacar que, a diferencia de Spinoza, este procedimiento sintético no agota lo que Tschirnhaus considera su método de descubrimiento -su ars inveniendi-. En efecto, en la Medicina Mentis, las principales reglas metodológicas consisten en una cierta combinación de análisis y de síntesis y están orientadas a la obtención de definiciones (Narváez 2013: 7). Con lo cual, su método es tanto analítico como sintético y ambos procesos están orientados al descubrimiento. Tschirnhaus, al igual que Spinoza, no distingue entre un método de descubrimiento y un método de justificación (ars inveniendi y ars demostrandi), aunque sí insiste en que su método consiste en unión del análisis y la síntesis. Con todo, a su juicio, es la síntesis el procedimiento que no solo permite descubrir nuevas verdades sino también garantizar su apodicticidad (Narváez 2013: 5).

Por último, también para el caso de Leibniz podemos afirmar que la definición es un punto de arranque en la búsqueda de la verdad. En el escrito titulado "Sobre la síntesis y el análisis universal, es decir, sobre el arte de descubrir y el arte de juzgar" (1683-1685), luego de explicar los tipos de conocimiento y los tipos de definición asociados a ellos, y en especial luego de hablar del conocimiento adecuado o intuitivo y de la definición causal, afirma:

A partir de estas ideas o definiciones, pues, pueden demostrarse todas las verdades, excepto las proposiciones idénticas, las que por su naturaleza es patente que son indemostrables. (Leibniz GPVII, 295) ${ }^{6}$.

En el mismo sentido, en las Meditaciones sobre el conocimiento la verdad $y$ las ideas, Leibniz señala que

[...] las definiciones nominales son insuficientes para una ciencia perfecta a no ser que se sepa con certeza por otro medio que la cosa definida es posible (Leibniz GP IV, 425).

\footnotetext{
${ }^{6}$ Salvo que indiquemos lo contrario, utilizaremos las traducciones de los escritos filosóficos de Leibniz editados por Ezequiel de Olaso.
} 
Podemos colegir de estos pasajes que, para Leibniz, las definiciones constituyen los pilares fundamentales a partir de los cuales se eleva deductivamente el edificio de la ciencia. Aunque, en ciertos casos, las definiciones nominales no conducen a la ciencia perfecta, existe, para Leibniz, otro tipo de definición que sí conduce a ella. En el apartado siguiente veremos cuáles. Por otra parte, en principio también puede colegirse de estos pasajes que la imagen que nos deja Leibniz del método es sintética. Por cierto, a partir de esta primera aproximación se ve que la función que le atribuye Leibniz a la definición se asemeja considerablemente a la visión que tenían Spinoza y Tschirnhaus. Se trata de que a partir de una definición dada puedan deducirse las propiedades del objeto definido (Couturat 1901: 191).

Cabe aclarar, no obstante, que esta imagen del método de Leibniz no es completa. Ciertamente, por un lado, hay también aspectos analíticos que deben ser tenidos en cuenta y, por otro, no se ha elucidado aún la cuestión de si se trata de un método de descubrimiento o de justificación. Ahora bien, dado que la concepción metodológica de Leibniz presenta múltiples aspectos y está diseminada en innumerables escritos a lo largo de un extenso corpus, solamente señalaremos algunos lineamientos fundamentales a fin de obtener una perspectiva plausible acerca de la función asignada a las definiciones.

En primer lugar, está fuera de discusión que tanto el análisis como la síntesis cumplen una función en la metodología leibniziana, sin embargo, la naturaleza de la relación es aún problemática. Una de las cuestiones más acuciantes, entre otras, es si debemos identificar al análisis y la síntesis con el método de demostración o de juicio (ars judicandi) y con el método de descubrimiento o invención (ars inveniendi), respectivamente, o si más bien debemos pensar que los procesos analíticos y sintéticos se presentan al mismo tiempo en uno y otro método. Esta cuestión ha dado lugar a diversas interpretaciones que se apoyan en ciertas diferencias que surgen de los propios escritos leibnizianos (Esquisabel 1999: 305-307). Quizá podamos resumir la cuestión de la siguiente manera. De entre las perspectivas que pueden encontrarse en los escritos leibnizianos se destaca una perspectiva "pragmática", orientada fundamentalmente a resolver problemas locales, y una perspectiva "teórica", orientada a la fundamentación absoluta de las ciencias. Esta última es la perspectiva que nos interesa aquí, pues, por una parte es la que está en juego en los pasajes que hemos citado arriba y, por otra, es la que coincide con el enfoque del concepto de método que estamos poniendo en consideración en los casos de Spinoza y Tschirnhaus, vale decir, un método que permita ampliar el conocimiento y fundamentarlo.

Ahora bien, desde esta perspectiva "teórica", el análisis tiene la función de llegar a los principios, a los fundamentos absolutos, mientras 
que la síntesis se ocupa de disponer el conocimiento de una manera axiomático-deductiva. Bajo esta perspectiva el método de descubrimiento y el método de justificación aparecen fundidos en un único bloque. Por un lado, el análisis conduce a los principios, permite descubrirlos y sacar a la luz su evidencia, por el otro, la síntesis permite descubrir de manera combinatorio nuevas verdades y, al mismo tiempo, justificarlas (Esquisabel 1999: 318). Es justamente en esta orientación donde la definición presenta una función fundamental, en tanto elemento central en cuanto que a partir de ella se obtienen nuevas verdades y al mismo tiempo se las demuestra deductivamente (Esquisabel 1999: 312-313).

En resumidas cuentas, tanto para Spinoza, como para Leibniz y Tschirnhaus, la definición aparece como el punto de partida de un método que presenta varias funciones al mismo tiempo, a saber, el descubrimiento de nuevas verdades, la fundamentación absoluta de las mismas y la organización sistemática del conocimiento. Se trata, en efecto, de una metodología orientada a alcanzar lo que Leibniz denomina "la ciencia perfecta". Así pues, aunque la definición nos remite, desde la antigüedad, a un método de carácter sintético y la síntesis - asociada con el mos geometricus - está ligada fuertemente a la fundamentación, sin embargo, en el caso de nuestros filósofos esta asociación es parcial, puesto que en sus concepciones el objetivo de fundamentación se entrecruza con el objetivo heurístico.

\section{La naturaleza de la definición}

$\coprod$ asta aquí hemos visto que existen notorias semejanzas en el no obstante, como veremos a continuación, la manera de entender la naturaleza de la definición por parte de estos tres filósofos nos conduce a una situación de mayor complejidad, ya que aquí encontramos ciertos elementos comunes pero también ciertas diferencias. En efecto, los tres filósofos están de acuerdo en que la definición perfecta debe ser una definición causal y que la misma se realiza en el ámbito del entendimiento, es decir, que consiste en una idea verdadera, pero aquí los caminos comienzan a bifurcarse. En lo que sigue veremos con más detalle cómo concibe cada uno dicha definición y hasta dónde llegan las similitudes y comienzan las diferencias.

Iniciaremos el recorrido examinando la concepción de Spinoza para luego poder trazar una comparación con las concepciones de Tschirnhaus y Leibniz. Según hemos mostrado en un trabajo previo, pueden distinguirse en las reflexiones y en la puesta en práctica metodológica del filósofo holandés tres tipos de definición: la definición nominal, la definición real y una mezcla 
de ambas. La definición nominal es aquella que indica solamente el modo en que se designa una cosa, es decir, hace referencia al nombre con el que se la designa. La definición real en cambio es aquella que indica la esencia de la cosa definida y, a diferencia de la nominal, requeriría demostración. Por otra parte, la definición nominal y real, que denominaremos mixta, es aquella que indica tanto el modo en que se nombra la cosa como la esencia de la cosa. Esta tipo de definición por su claridad y distinción no requeriría de una demostración para garantizar su verdad. Esta concepción, tal como hemos indicado, acusa la influencia de ciertos lineamientos de la Lógica de Port Royal. Aunque es claro que la teoría de la definición de Spinoza presenta muchas características originales (Narváez 2019: 70 y ss.).

Spinoza se distancia de los filósofos de Port Royal en el modo en que concibe a la definición real y a la definición mixta. En efecto, mientras que Arnauld y Nicole siguen la concepción aristotélica que define a través del género y la diferencia específica, Spinoza considera que la definición real (mixta) debe mostrar la causa del objeto definido. Así, para él, hay tres requisitos que debe cumplir la definición perfecta. El primero de ellos consiste en que la definición sea capaz de poner ante los ojos de la mente la esencia de la cosa definida. No obstante, para Spinoza, conocer la esencia no es conocer el género y la diferencia específica sino conocer la causa de su generación. Un segundo requisito que hace a la buena definición es que puedan deducirse de ella todas las propiedades de la cosa. Por último, un tercer requisito es que la definición sea clara y distinta para que de ese modo no quepa duda de la verdad de la definición. Esto es, la definición pone ante los ojos de la mente una idea adecuada y verdadera, captada por la mente de una manera intuitiva ${ }^{7}$.

Tenemos dos casos paradigmáticos para entender en qué consiste la concepción de Spinoza, uno en el ámbito de la geometría y otro en el de la metafisica. El primero aparece en el Tratado de la reforma del entendimiento y se refiere a la definición de círculo. Dice lo siguiente:

La definición deberá, como hemos dicho, comprender su causa próxima. El círculo, por ejemplo, conforme a esta regla, debería ser definido diciendo que es la figura que es descrita por una línea cualquiera, uno de cuyos extremos es fijo y el otro móvil, pues esta definición incluye claramente la causa próxima. (Spinoza TIE $\$ 96$, G II 35)

\footnotetext{
${ }^{7}$ Sobre el concepto de intuición que está en juego aquí véase Narváez 2017.
} 
El segundo aparece en la correspondencia con Tschirnhaus y se refiere a la definición de Dios dada en la Ética. Allí puede leerse:

[...] cuando defino a Dios como el ser sumamente perfecto, como esa definición no expresa la causa eficiente (pues entiendo por causa eficiente tanto la interna como la externa), no podré extraer de ahí todas las propiedades de Dios. En cambio, cuando defino a Dios como el Ser, etc., vea la definición VI de la parte I de la Ética [sí podré]. (Spinoza Ep.60, G IV 270-271)

Como es bien conocido la definición en cuestión es la siguiente:

Por Dios entiendo el ser absolutamente infinito, es decir, la sustancia que consta de infinitos atributos, cada uno de los cuales expresa una esencia eterna e infinita. (Spinoza E I def. 6, G II 44)

Tenemos aquí un claro ejemplo de definición mixta, al utilizar la cláusula "por x... entiendo...", Spinoza adopta el formato de la definición nominal, sin embargo, luego expresa la esencia o la causa de la cosa definida. Esto es, aquello que si se pone se da también la cosa y si se quita se quita la cosa. $\mathrm{O}$, en otros términos, aquello sin lo cual la cosa no puede ser ni ser pensada (Spinoza E II def. 2, G II 44). De este modo, la definición de Dios de la Ética, tanto como la de círculo del Tratado, cumpliría con los requisitos que debe cumplir la definición perfecta.

Es muy importante no perder de vista que la aclaración acerca de la naturaleza de este tipo de definición fue realizada por Spinoza justamente a instancias de Tschirnhaus, quien en ese momento -1676- se encuentra en París y posee en sus manos una copia de la Ética, o al menos de una parte de ella. El mismo momento y lugar en el que dicho filósofo entabla amistad con Leibniz y conversa con él sobre la filosofia de Spinoza.

Pues bien, cerca de diez años después de recibir dicha carta Tschirnhaus publica su tratado sobre el método. No encontramos allí las diferentes clases de definiciones que pueden rastrearse en los escritos de Spinoza, sino que Tschirnhaus parece concebir un único tipo de definición. La misma, no obstante, se acerca notablemente a la definición perfecta de Spinoza. En efecto, notamos que no hay en la Medicina Mentis ninguna mención de la clásica distinción entre definiciones nominales y reales. Sin embargo, la caracterización que veremos a continuación nos lleva a pensar que Tschirnhaus da por descontado que la definición perfecta es una definición real (definitio realis). Tal como podemos leer allí, 
[...] Si una definición es lo que es concebido de una cosa en primer lugar, y lo que excluye que alguna cosa pueda ser concebida antes que ella y si, por otra parte, nada puede ser concebido de una cosa que sea anterior a su generación, entonces, en verdad, toda definición legítima o buena, comprenderá la generación de la cosa. (Tschirnhaus $M M, 92-93$ )

\section{Dicho en otros términos:}

[...] si la definición que Euclides ha dado de la proporcionalidad fuera buena, es decir, si ella comprendiera la generación de la proporción, no habría habido, sin duda, tanta discusión sobre este asunto. En cuanto a los moralistas, si sus definiciones fueran auténticas, las pasiones del alma serían provocadas en nosotros si se dieran las condiciones requeridas para tal efecto en sus definiciones. Así, si la definición que explica la naturaleza de la risa fuera buena, haríamos reír también a otras personas toda vez que las condiciones de la risa indicadas por esta misma definición se encontraran reunidas. (Tschirnhaus $M M, 93$ )

Como puede apreciarse, la buena definición de Tschirnhaus es causal como la de Spinoza, entendiendo por causa aquellas condiciones tales que, si se dan, debe darse necesariamente la cosa de las cuales son causa. No entraremos aquí en la discusión respecto del tipo de causalidad que está en juego en la Medicina Mentis; lo cierto es que la visión que presenta Tschirnhaus a través de estos ejemplos hace suponer que admite la posibilidad de tales definiciones en diferentes ámbitos del conocimiento, desde la geometría hasta la ética.

Otro rasgo de la definición que se vislumbra en estos ejemplos se refiere a la cuestión de la certeza y la verdad de la definición. Por cierto,Tschirnhaus nos da a entender que si la definición es buena, esto es, si muestra la generación de la cosa, se eliminan los malentendidos y las discusiones, de donde se sigue que la buena definición es acompañada por la evidencia de lo que se define. Este punto se aclara aún más si tenemos en cuenta que el acto de definir es al mismo tiempo un acto por el cual el entendimiento concibe, esto es, forma un concepto. De manera que, también para Tschirnhaus, la definición genética manifiesta una idea verdadera, entendida, al igual que Spinoza, como una acción del entendimiento. Según nuestro filósofo, "es claro que una vez dada una tal definición, no podría subsistir ninguna duda en cuanto a la certeza de la cosa definida" (Tschirnhaus0 MM, 94).

Nos detendremos brevemente sobre este asunto ya que es un punto clave de la concepción metodológica y epistemológica del filósofo sajón y, por ello, es indispensable para completar la imagen que estamos delineado de su teoría de la definición. Para el autor de la Medicina Mentis, una idea 
o una afirmación falsa es una idea o una afirmación que no podemos concebir, mientras que es verdadera aquella idea o afirmación que sí podemos concebir. Tschirnhaus considera que este es el criterio básico de la certeza humana a través del cual se puede distinguir lo verdadera de lo falso y levantar a partir de allí el edificio del conocimiento. Ahora bien, ¿qué entiende nuestro filósofo por "concebir"? Tschirnhaus indica sucintamente que "concebimos" aquello que está en nuestro poder de pensar y aquello que no conduce a una contradicción, esto es, aquellas ideas que podemos unir de una manera clara y plenamente consciente. Por ejemplo, no podemos pensar, salvo con meras palabras, que el todo es menor que la parte, pero si podemos pensar la afirmación opuesta, por lo tanto, esto nos indica claramente cuál afirmación es verdadera y cuál es falsa (Tschirnhaus $M M, 69$ ).

En la manera en la que Tschirnhaus entiende la concebibilidad está presente la concepción de la idea como un cierto compuesto de ideas simples, así como también la exigencia de que dichos elementos deben ser compatibles entre sí. Esto puede observarse en las reglas para la obtención de las definiciones, en las que Tschirnhaus da algunas precisiones acerca de cómo llegar a concebir una cosa. Como hemos visto, de lo que se trata es de indicar las causas, es decir, las condiciones necesarias por las cuales lo definido existe. Dicho muy esquemáticamente, para ello, es preciso hallar mediante el análisis, en primer lugar, los géneros máximos o últimos, luego los elementos irreductibles dentro de cada uno de los géneros máximos, distinguiendo los fijos por un lado y los móviles por el otro. Finalmente, combinar dichos elementos de todas las maneras posibles hasta que surjan de dichas combinaciones las definiciones, esto es, las cosas posibles (MM, 96-97; 106-107). El ejemplo geométrico utilizado por Tschirnhaus para clarificar este punto es la conocida definición de círculo, que consiste en un construir un círculo haciendo girar un segmento manteniendo uno de sus puntos fijos (MM, 109).

Como puede observarse, el lenguaje y la concepción epistemológica general presentada por Tschirnhaus guardan una estrecha similitud con la concepción spinoziana. Ciertamente, vemos que la definición genética se acopla con el concepto de idea, entendido como una actividad del entendimiento, que no debe confundirse con las imágenes pasivas de la imaginación. Utilizando un lenguaje muy reminiscente del lenguaje de Spinoza, Tschirnhaus señala que esta actividad se convierte en la norma de la verdad y la falsedad que llevamos dentro, como una luz que distingue en sí misma la claridad y la oscuridad. En la misma línea, nuestro filósofo señala que de lo verdadero se sigue lo verdadero, mientras que de lo falso se desprenden cosas contradictorias. En la misma dirección de pensamiento, Tschirnhaus vincula la dicotomía entre el ser y la verdad y el no ser y la falsedad, con la 
dicotomía entre lo posible y lo imposible, al señalar que entre el ser y el no ser no existe otra diferencia que entre lo posible y lo imposible, o lo concebible y lo inconcebible. ${ }^{8}$

De este modo, las definiciones se convierten en la herramienta principal del método, ya que, al indicar las causas del objeto definido, construyen su naturaleza o esencia $(M M, 94)$, mostrando al mismo tiempo su concebibilidad $^{9}$ y su verdad. Por otra parte, en la medida en que se definen los objetos fundamentales de una ciencia se establecen las bases sobre las que se descubren y se justifican el resto de las verdades, esto es, las propiedades de los objetos definidos. En otras palabras, se establecen las bases sobre las que se asienta todo el resto del edificio de la ciencia.

Por último, cabe agregar una cuestión no menor referente a las funciones que nuestro filósofo le atribuye a la misma. La función principal de acuerdo a lo expresado anteriormente es establecer los conceptos fundamentales de una ciencia, esto es, concebir la esencia de los mismos a través de su generación. Sin embargo, es claro que Tschirnhaus también le ha atribuido a la definición genética la función de indicar el uso correcto de los nombres utilizados. En efecto, según señala en un capítulo de la Medicina Mentis dedicado a eliminar los errores de la imaginación en la búsqueda de la verdad, las definiciones cumplen una función importante en la medida en que se utilizan para distinguir adecuadamente los términos que presentan significados diferentes. En este sentido, las definiciones son una ayuda o un remedio para evitar los errores causados por el uso inapropiado de la imaginación. Así, definir correctamente es uno de los procedimientos que, para nuestro filósofo, permiten subordinar la imaginación al entendimiento. ${ }^{10}$ En consecuencia, es claro que Tschirnhaus también le atribuye a la definición una función lingüística, de modo que la buena definición debe ser considerada tanto una definición real como una definición nominal. ${ }^{11}$

Hemos delineado hasta aquí los rasgos más salientes de la concepción de la definición que Tschirnhaus expuso en su Medicina Mentis, sobre la que comenzó a reflexionar en diálogo con Spinoza, unos diez años antes, mientras se encontraba en Paris. Como sabemos, allí también se encontraba

\footnotetext{
${ }^{8}$ Véanse Tschirnhaus MM, 69-70 y Van Peursen 1993: 405-406

9 Tomamos este concepto de Campo, quien utiliza el término latino "conceptibilitas" o el italiano "conceptibilità" para referirse al criterio propuesto por Tschirnhaus (Campo 1939: 24 y ss.).

${ }^{10}$ Véanse Tschirnhaus $M M, 175$ y Narváez 2013: 9

${ }^{11}$ La definición genética no se opone a la definición real ni a la definición nominal como parece entender Campo(1939: 30).
} 
Leibniz con quien entabló una relación de amistad que les permitió a ambos mantener un provechoso intercambio intelectual. De entre todos los temas sobre los que conversaron seguramente el tema de la definición fue uno de los más importantes e indudablemente las conversaciones sobre la definición incluían el tratamiento de la concepción spinoziana, que Tschirnhaus conocía tanto por su aplicación en la Ética como por las aclaraciones que recibía a través del diálogo epistolar que mantenía con el mismo Spinoza. Evidentemente estas conversaciones y el posterior encuentro personal que mantuvo Leibniz con Spinoza dejaron sus huellas en el filósofo de Leipzig. Por ello, a continuación examinaremos los puntos más importantes de su concepción de la definición a fin de determinar con más precisión el alcance del efecto de la teoría de Spinoza.

Es importante notar que, si bien Leibniz reflexiona sobre el tema metodológico desde su juventud, realizando diferentes esbozos metodológicos, su teoría de la definición aparece de una manera más o menos acabada recién en un escrito posterior al período de París; nos referimos a "Sobre la síntesis y el análisis universal, es decir, sobre el arte de descubrir y el arte de juzgar", confeccionado alrededor de $1683-1685 .{ }^{12}$ Este aspecto histórico del pensamiento de Leibniz representa un primer indicio de que la discusión con Tschirnhaus y el conocimiento de Spinoza marcan un momento importante en la evolución del pensamiento leibniziano en lo que respecta a cuestiones metodológicas. Este punto también nos lleva a pensar que, con respecto a la originalidad de las concepciones metodológicas, Leibniz le lleva cierta ventaja al autor de la Medicina Mentis, no solo por la mencionada prioridad temporal del escrito mencionado con respecto a la Medicina Mentis, sino también porque al parecer, Leibniz consideró que su amigo sajón lo había plagiado en este punto (Wurtz 1988: 202-203) ${ }^{13}$.

Sea como fuere, su concepción incluye una distinción que no encontramos en el autor de la Medicina Mentis. Ciertamente, Leibniz distingue claramente dos tipos de definición, la definición nominal y la definición real.

\footnotetext{
${ }^{12}$ Nos ha sido señalado en una charla personal por el Prof. Esquisabel que no hay indicios de la teoría de la definición causal en el período temprano.

${ }^{13}$ En una conocida carta a Tschirnhaus, Leibniz menciona lo fundamental de su teoría de la definición, esto es, que la definición adecuada y perfecta es aquella que no deja dudas acerca de la posibilidad de la cosa definida. También señala que hay definiciones que permiten distinguir la cosa a través de la indicación de alguna de sus propiedades recíprocas pero estas no serían la mejor forma de definir (GM IV, 451 y ss.). Claramente está en germen aquí la distinción entre definición nominal y real. No obstante, ello no parece ser una prueba definitiva para admitir el plagio por parte de Tschirnhaus.
}

MARIO A. NARVÁEZ - La teoría de la definición en Leibniz, Spinoza y Tschirnhaus I 109-129 
Tal como explica en "Sobre la síntesis y el análisis universal", la definición nominal es aquella que, mediante la mención de al menos una de las notas o de los requisitos del objeto definido, permite diferenciar la cosa definida de las demás (Leibniz GPVII, 293). Dicho concepto lo toma Leibniz de la tradición escolástica aunque también pudo haberlo encontrado en Hobbes, quien identifica la causa sine que non -o también la causa íntegra- con la suma de los requisitos. Leibniz, como señala Velarde, utiliza dicho concepto muy tempranamente -incluso antes de haber elaborado su teoría de la definición causal- en un sentido cercano al de Hobbes y vinculado con el concepto de razón suficiente (Velarde 2015: 2-4.).

Las definiciones reales, en cambio, deben enumerar los requisitos que muestran que la cosa definida es posible. Para ello, señala Leibniz, es necesario o bien presentar el modo de generar la cosa, o bien descomponer la cosa en las nociones primitivas últimas que la componen y que son inteligibles por sí mismas (GPVII, 295). Cabe destacar además que, en ambos casos, las definiciones genéticas "envuelven una causa próxima", pues, si bien la descomposición muestra el modo en que se genera la cosa, la suma de los requisitos constituye para Leibniz su causa/razón suficiente (Velarde 2015: 2-4). De este modo, se obtiene una definición real "perfectísima”, en la que es "inmediatamente patente la posibilidad" de lo definido. En el caso de que se llegue a la descomposición de las "nociones primitivas últimas", el conocimiento que se obtiene aquí es "adecuado o intuitivo" (GPVII, 295). Cabe agregar, que un ejemplo que utiliza Leibniz para ilustrar esto es la definición genética de círculo -el mismo ejemplo utilizado por Spinoza y Tschirnhaus-, esto es, el movimiento de una línea recta manteniendo fijo uno de sus extremos (GPVII, 295).

Cabe señalar, por otra parte, que el tema de la definición es tratado por Leibniz en varios lugares, pero en términos generales la concepción se mantiene constante. En el famoso escrito "Meditaciones sobre el conocimiento, la verdad y las ideas", compuesto unos años más tarde (noviembre de 1684), la naturaleza de la definición aparece asociada de una manera más estrecha a la temática epistemológica. Aquí la definición nominal se vincula a lo que Leibniz denomina conocimiento inadecuado, pues aunque es una noción distinta, es decir, aquella noción que nos permite distinguir una cosa de otras cosas parecidas mediante la "enumeración de las notas suficientes", no permite saber si entre dichas notas no existe una contradicción, pues las mismas no se pueden conocer de manera clara (GPVII, 423). La definición real, por su parte, está asociada al conocimiento adecuado, pues al permitir descomponer la noción en sus requisitos últimos, es decir, al llevar el análisis hasta las nociones primitivas que se conocen claramente, permite también comprobar que no existe contradicción entre tales nociones y, por ello, que 
la cosa es posible (GP IV, 424-425). ${ }^{14} \mathrm{Tal}$ conocimiento analítico se obtiene a priori, aunque la posibilidad de la cosa también se puede conocer a posteriori, esto es, experimentando su existencia en acto a través de los sentidos. Cabe mencionar, además, que en el Discurso de Metafísica, Leibniz agrega que la definición real a priori, en cuanto llega al conocimiento de las nociones primitivas últimas de la cosa, conduce al conocimiento de la esencia de la cosa definida (GP IV, 450).

Por último, un aspecto sumamente importante a los fines de nuestra comparación con las teorías de la definición de Spinoza y Tschirnhaus, es que también en la teoría leibniziana el concepto de definición guarda una estrecha relación con el concepto de idea. De igual modo que en dichos filósofos, la temática metodológica y la temática epistemológica confluyen en la definición. Ciertamente, Leibniz, como aquellos, asocia la noción posible con la idea verdadera y la noción contradictoria con la idea falsa, de modo que la definición real, en tanto que muestra la posibilidad de la cosa, es el vehículo que permite al filósofo formar una idea verdadera. En efecto, Leibniz afirma explícitamente en sus meditaciones que una idea es verdadera "cuando la noción es posible y falsa cuando encierra contradicción” (GP IV, 425).

Ahora bien, aunque se trata de una similitud importante, debemos considerarla con cierto detenimiento ya que el concepto de idea en Leibniz tiene ciertas particularidades que lo distancian del concepto spinoziano. Por ello, necesitamos previamente esclarecer dicho concepto, aunque más no sea de un modo esquemático, para luego determinar con mayor precisión su vínculo con la teoría de la definición. Leibniz se ocupó de la problemática de las ideas examinando las concepciones de los filósofos cartesianos que intentaban clarificar el concepto de idea que Descartes había dejado en gran medida envuelto en un velo de ambigüiedad, pero también las posiciones materialistas que rechazaban de plano la posición cartesiana (Jolley 1990: 132 y ss.). En un brevísimo escrito denominado “¿Qué es idea?”, Leibniz presenta su característica visión de las ideas. En primer lugar, se enfrenta claramente al materialismo, distinguiendo la imaginación del entendimiento, "las huellas en el cerebro no son ideas", las ideas están en nuestra mente (GPVII, 263). En segundo lugar, Leibniz toma posición frente a los cartesianos, aquí particularmente frente a la concepción de Arnauld o de Spinoza, "la idea

\footnotetext{
${ }^{14}$ Para un interesante análisis sobre esta clasificación leibniziana en paralelo con la clasificación de los géneros de conocimiento del TIE en el que pueden verse interesantes similitudes, véase Leinkauf 2014: 318-319. Nos interesa señalar que de allí surge que la definición genética corresponde en ambos casos al género supremo de conocimiento. Esto es, Leibniz parece coincidir con Spinoza en cuanto a las características fundamentales de dicho conocimiento.
}

MARIO A. NARVÁEZ - La teoría de la definición en Leibniz, Spinoza y Tschirnhaus I 109-129 
no consiste en un acto del pensamiento sino en una facultad". Esto implica que tenemos una idea aunque no estemos pensando en ella en el momento presente, siempre y cuando podamos pensar en ella si así lo deseamos (GP VII, 263). Por otra parte, Leibniz indica en el Discurso de Metafísica que las ideas representan las naturalezas o esencias de las cosas que están en nuestra alma para ser descubiertas (GPVII, 451). Esta concepción no contradice la concepción de Arnauld o de Spinoza en cuanto a que la idea es un acto; es, más bien, una versión matizada, por así decir. En efecto, en la medida en que la idea es pensada en el presente, es sin dudas un acto del entendimiento. Así pues, es claro que la definición real, en cuanto permite mostrar la posibilidad de la cosa definida, es la expresión en acto de una idea presente en el alma humana, la actualización de la misma. En consecuencia, en la medida en que alguien comprende la definición, realiza una acción del entendimiento. Como señala Jolley, la teoría de la idea de Leibniz intenta conciliar la posición de Malebranche y la de Arnauld, haciendo una especie de síntesis (Jolley 1990: 135). Hay muchos aspectos de esta teoría que pasamos por alto, así como también una discusión de los puntos de vista interpretativos sobre las no pocas cuestiones ambiguas en los textos leibnizianos. Nos parece suficiente, dada la perspectiva de este trabajo y el espacio del que disponemos, saber que la idea en cuanto se actualiza en una definición real es una actividad del entendimiento, de un modo similar a como lo es en el caso de Spinoza o Tschirnhaus. De este modo, la diferenciación que establece Leibniz, no apunta a rechazar la concepción de la idea como acto, sino más bien a mostrar que no todo acto del entendimiento es una idea. En efecto, puede suceder que algo que en principio se concibe como una idea, al ser un conocimiento no adecuado, esto es, al no conocerse sus componentes últimos, resulte una noción contradictoria. En sus Meditaciones, Leibniz pone como ejemplo la idea del movimiento más veloz, idea que podemos pensar pero que en cuanto se la examina en sus elementos se descubre una contradicción y por lo tanto, no es más que una idea solo en apariencia, de donde se sigue que las conclusiones extraídas de dicha noción también pueden ser falsas. Leibniz advierte a los cartesianos que lo mismo puede ocurrir con la idea de Dios si no se muestra su posibilidad a través de una definición genética correctamente formulada (GPVII, 424). Pues, la definición de Dios como "causa sui" dada por Spinoza no muestra la posibilidad de la noción misma de Dios, es decir, no muestra cuáles son sus componentes últimos y por ello no se puede establecer si son combinables entre sí.

Para finalizar, cabe aclarar que Leibniz es muy prudente respecto a la posibilidad de que la inteligencia humana llegue a obtener definiciones reales de todas las cosas. En efecto, el filósofo de Leipzig es consciente de que la ciencia humana es incapaz de llegar a tales definiciones en todos los 
dominios del conocimiento, sin embargo, ello no impide que usualmente pueda progresar hacia la verdad a través de definiciones nominales, a través de operaciones simbólicas (GPVII, 423). Así, la ciencia perfecta para la cual es indispensable contar con definiciones reales aparece en cierto modo como un ideal regulativo.

\section{Conclusiones}

T eamos si podemos sacar algunas conclusiones preliminares a partir de lo expuesto hasta aquí. Salta a la vista, en primer lugar, que la teoría de la definición ocupa un papel preponderante en los tres autores. En los tres casos la definición presenta características similares tanto en su naturaleza como en su función. Así, los tres filósofos coinciden en que la definición perfecta es una definición causal, que muestra la esencia de la cosa definida y presenta una idea adecuada de la misma. En cuanto a su función, la definición es el punto de partida de un método orientado a descubrir y a demostrar nuevas verdades. Por otra parte, la definición sirve para fijar el significado de las palabras y evitar los errores lingüísticos. En Spinoza y en Tschirnhaus la clásica distinción entre definición nominal y real se desdibuja y la definición causal conjuga esas dos funciones. Por ello, las definiciones genéticas en la medida en que no requieren demostración pueden ser los principios de las demostraciones. En el caso de Leibniz, la clásica distinción se mantiene, aunque la definición real en la medida en que no requiere demostración sino que es un principio, adquiere, por así decir, cierto carácter nominal. En efecto, si bien Leibniz mantiene la distinción entre la definición nominal y real, se distancia de ella, en cuanto que, para él, las definiciones reales no requieren demostración.

Pues bien, detrás del modo en que cada uno de los filósofos entiende la definición está también el modo en que conciben la naturaleza de la idea y también la naturaleza de la causalidad. En el presente trabajo nos hemos limitado a examinar brevemente el vínculo entre el concepto de definición y el concepto de idea, dejando para otra ocasión la cuestión de la causalidad. A nuestro modo de ver las concepciones de Leibniz y de Tschirnhaus en relación a la naturaleza de la idea no se oponen a la concepción spinoziana, sino que más bien intentan llenar las lagunas que dejó el filósofo holandés tanto como superar los escollos que presenta el concepto de idea en el cartesianismo en general.

Ciertamente, la función de la definición genética, en tanto pilar metodológico, no es una concepción original del pensamiento spinoziano, lo propio de dicho pensamiento en lo referente a la metodología es más bien 
el haber combinado ciertos aspectos metodológicos tomados fundamentalmente de Hobbes con otros tomados del cartesianismo. Es aquí donde su influencia se hace sentir tanto en la metodología de Tschirnhaus como de Leibniz. En efecto, sus proyectos metodológicos no contrarían el marco teórico spinoziano sino que lo amplían. Así, vemos que una complejización en la teoría del conocimiento conlleva al mismo tiempo una complejización metodológica.

Por otra parte, también es interesante constatar que el tema de la definición es muy importante para examinar el intercambio intelectual entre Leibniz y Tschirnhaus. Si bien es una cuestión que merece abordarse con mayor detenimiento, a través de nuestro breve recorrido podemos observar que dichos filósofos divergen en una cuestión central, a saber: la función de las definiciones nominales. Es aquí donde Tschirnhaus parece mantenerse más cercano a Spinoza que lo que lo hace Leibniz. Ciertamente, al asignar una función a las definiciones nominales, Leibniz está haciendo una concesión al conocimiento imperfecto, a una ciencia incompleta y acorde a las posibilidades humanas. Tschirnhaus, por su parte, parece considerar que solo vale la pena buscar definiciones genéticas, es decir, aquellas en las que se manifiesta la esencia de la cosa de una manera completa y a priori. En consecuencia, su exigencia epistemológica es mucho mayor.

Finalmente, cabe destacar que ambos filósofos coinciden en que ciertos procedimientos analíticos tienen una función fundamental en la obtención de las definiciones. Así como también en la complementariedad entre los procedimientos analíticos y sintéticos. Es preciso, sin embargo, examinar con mayor detalle el modo en que cada uno entiende estos procedimientos, como también la función de las definiciones en el proceso deductivo. Pero es una cuestión que queda para una investigación ulterior. ${ }^{15}$

\section{BIBLIOGRAFÍA}

Arnauld, A y Nicole, P. (1663), La Logique ou L'Art de Penser (Paris).

Arnauld, A. y Nicole, P. (Logique), La Lógica o el Arte de Pensar, prólogo, traducción y notas de G. Quintás Alonso (Madrid: Alfaguara, 1987).

Arndt, W. (1971), Methodo Scientifica Pertractatum (Berlin-New York: Walter de Gruyter).

${ }^{15}$ El presente trabajo fue realizado en el marco del proyecto de investigación PICT 20170506 de la ANPCyT (Argentina), titulado "La Ciencia General de Leibniz como fundamentación de las ciencias: lógica, ontología y filosofía natural" 
Cabañas, L. y Esquisabel, M. O. (2014) (eds.), Leibniz frente a Spinoza: una interpretación panorámica (Granada: Comares).

Campo, M. (1939), Cristiano Wolff e il Razionalismo Precritico (Milano: Societá edritice vita e pensiero).

Couturat, L. (1901), La Logique de Leibniz d'après des Documents inédits (Paris: Felix Alcan)

De Angelis, E. (1968), "El Método Geométrico de Descartes a Spinoza”, Tarea, 1:25-47.

Esquisabel, O. M. (1999), "Perspectivas leibnizianas sobre el Análisis y la Síntesis", Theoria, 14 (2): 303-329.

Goldenbaum, U. (2014), "La fascinación de Leibniz por Spinoza”, en L. Cabañas y M. O. Esquisabel (2014) (eds.), Leibniz frente a Spinoza: una interpretación panorámica (Granada: Comares, 31-50).

Joachim, H. (1901), A Study of the Ethics of Spinoza (Oxford: Clarendon Press).

Jolley, N. (1990), The Light of the Soul: Theory of Ideas in Descartes, Malebranche and Leibniz (Oxford: Oxford University Press).

Kulstad, M. A. (2014), “Leibniz, Spinoza, y Tschirnhaus: Metafísica 'a tres manos', 16751676”, en L. Cabañas y M. O. Esquisabel (2014) (eds.), Leibniz frente a Spinoza: una interpretación panorámica (Granada: Comares: 51-70).

Laerke, M. (2008), Leibniz lecteur de Spinoza : la genèse d'une opposition complexe (Paris: Honoré Champion).

Leibniz, G. W. (GM), Die matematische Schriften, ed. de C. I. Gerhardt, 7 vols. (Berlín, Halle, 1849-1863; reimp. Hildesheim, 1962).

Leibniz, G. W. (GP), Die Philosophischen Schriften, ed. de C. I. Gerhardt, 7 vols. (Berlín, Halle, 1875-1890; reimp. Hildesheim: 1960-1961)

Leibniz, G. W. (1989), A Selection of Philosophical Papers and Letters, translated and edited, with and Introduction by L. E. Loemker (Dordrecht/Boston/London: Kluwer Academic Publishers).

Leinkauf, T. (2014), “El Tratado de Leibniz 'Meditationes de Cognitione, Veritate et Ideis' de 1684: Una discusión en torno al 'Tratactus de Intellectus Emendatione' de Baruch Spinoza”, en L. Cabañas y M. O. Esquisabel (2014) (eds.), Leibniz frente a Spinoza: una interpretación panorámica (Granada: Comares: 51-70).

Narváez, M.A. (2013), "El ars inveniendi de Tschirnhaus: un híbrido metodológico y un sustituto del lenguaje universal", Actas de las IX Jornadas de Investigación en Filosofía, 28 al 30 de agosto de 2013, La Plata, Argentina. URL = <http://jornadasfilo. fahce.unlp.edu.ar/ix-jornadas/actas-2013/a54.pdf>.

Narváez, M. A. (2017), "Uno intuitu videmus: la naturaleza del conocimiento intuitivo en Spinoza a la luz de Descartes", Revista Latinoamericana de Filosofia, 43 (2): 159-181.

Narváez, M. A. (2019), "La naturaleza y la función de las definiciones en la Ética de Spinoza", Revista Anales del Seminario de Historia de la Filosofía, 36 (1): 65-85.

Spinoza, B. (E), Ética demostrada según el orden geométrico, traducción de A. Domínguez (Madrid:Trotta, 2000). 
Spinoza, B. (Ep.), Correspondencia, traducción de A. Domínguez (Madrid: Alianza, 1998).

Spinoza, B. (TIE), Tratado de la reforma del entendimiento, traducción de A. Domínguez (Madrid: Alianza, 1988).

Spinoza, B. (1925), Opera, edición de C. Gebhardt, 4 vols. (Heidelberg: C. Winter, reed. 1972).

Tschirnaus, W. E. von $(M M)$, Medicina de L'Esprit ou préceptes généraux de l'art de découvrir, introduction, traduction, notes et appendices par J.-P. Wurtz (Paris: Ophrys).

Tschirnhaus, W. E. von (1695), Medicina Mentis, sive artis inveniendi precepta generalia, Editio nova, auctior et correctior cum praefatione autoris (Lepzig: Thomas Frisch).

Van Peursen, C. A. (1993), "E. W. Von Tschirnhaus and the Ars Inveniendi", Journal of History of Ideas, 54 (1): 395-410.

Velarde Lombraña, J. (2015), La teoría de la definición de Leibniz (Granada: Comares).

Wurtz, J.-P. (1988), "Über einige offene oder strittige, die Medicina Mentis von Tschirnhaus betreffenden Fragen”, Studia Leibinitana, 20 (2): 190-211.

Recibido: 24-07-2019; aceptado: 20-10-2019 